\title{
Erratum to: Exploring functional data analysis and wavelet principal component analysis on ecstasy (MDMA) wastewater data
}

\author{
Stefania Salvatore ${ }^{1 *}$, Jørgen G. Bramness ${ }^{1}$ and Jo Røislien ${ }^{1,2}$
}

\section{Erratum}

After publication of the original article [1], it came to the authors' attention that there were errors in Fig. 3, Fig. 4 and Additional file 1: Figure S1.

In each Figure, panels $\mathrm{A}, \mathrm{B}$ and $\mathrm{C}$ are not correct (but panels $\mathrm{D}, \mathrm{E}$ and $\mathrm{F}$ are). This error was due to a mistake in the last stages of the submission process while adjusting the Figures' size to fit the journal's requirements. This error does not impact the results, discussion and conclusions of the paper.

The correct version of the affected Figures are published in this erratum.

\section{Additional file}

Additional file 1: Figure S1. (A) Functional principal component analysis using Fourier basis functions with no smoothing. (B) Functional principal component analysis using Fourier basis functions with common -optimal smoothing. (C) Functional principal component analysis using Fourier basis functions with individual-optimal smoothing. (D) Functional principal component analysis using B-spline basis functions with no smoothing. (E) Functional principal component analysis using B-spline basis functions with common-optimal smoothing. (F) Functional principal component analysis using B-spline basis functions with individual-optimal smoothing. (PDF $27 \mathrm{~kb}$ )

\section{Author details}

${ }^{1}$ Norwegian Centre for Addiction Research, University of Oslo, Oslo, Norway. ${ }^{2}$ Oslo Centre for Biostatistics and Epidemiology, Institute of Basic Medical Sciences, Oslo, Norway.

Published online: 23 February 2017

\section{References}

1. Salvatore S, Bramness JG, Røislien J. Exploring functional data analysis and wavelet principal component analysis on ecstasy (MDMA) wastewater data. BMC Med Res Methodol. 2016;16:81. doi:10.1186/s12874-016-0179-2.

\footnotetext{
* Correspondence: stefania.salvatore@medisin.uio.no

${ }^{1}$ Norwegian Centre for Addiction Research, University of Oslo, Oslo, Norway
} 
A Functional principal component analysis using Fourier basis functions with no smoothing and 1000 bootstrapping samples
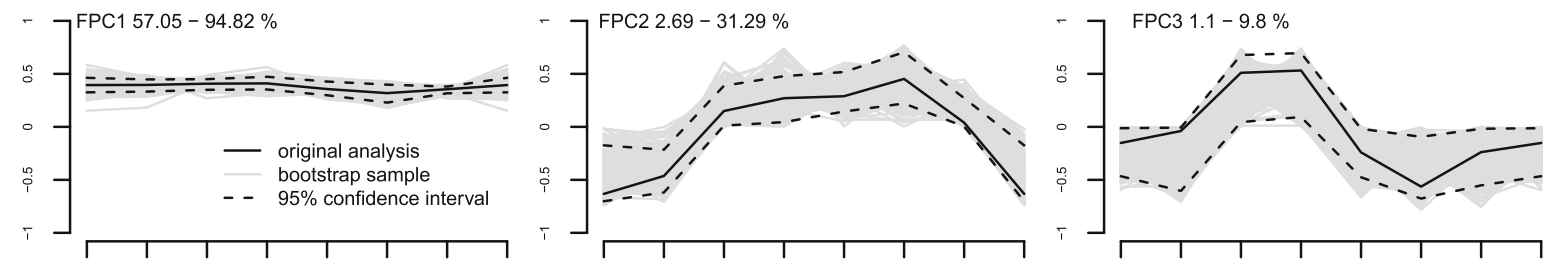

Wed Thu Fri Sat Sun Mon Tue Wed Wed Thu Fri Sat sun Mon Tue Wed Wed thu Fri Sat Sun Mon Tue Wed

B Functional principal component analysis using Fourier basis functions with common-optimal smoothing and 1000 bootstrapping samples
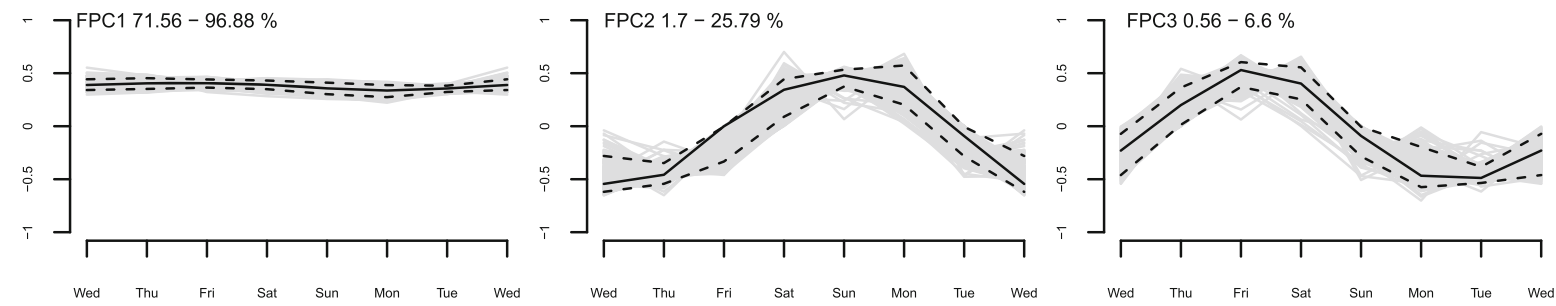

C Functional principal component analysis using Fourier basis functions with individual-optimal smoothing and 1000 bootstrapping samples
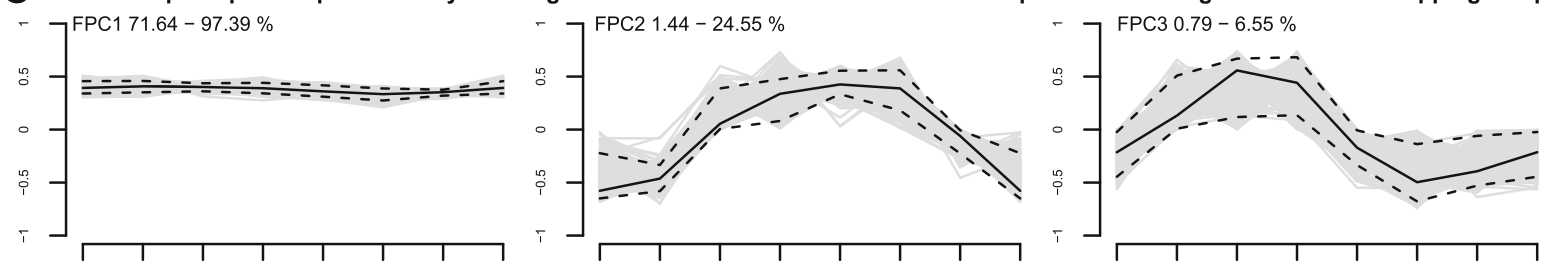

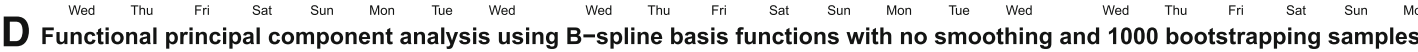

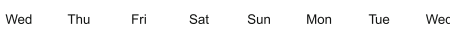

Wed Thu Fri Sat Sun Mon Tue Wed
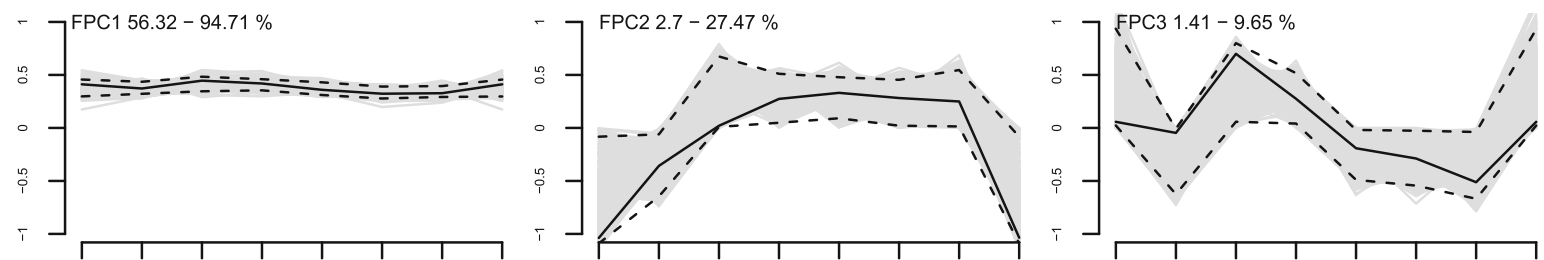

Wed Thu Fri Sat Sun Mon Tue Wed

Wed Thu Fri Sat Sun Mon Tue Wed

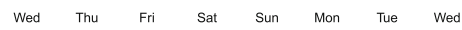

E Functional principal component analysis using B-spline basis functions with common-optimal smoothing and 1000 bootstrapping samples
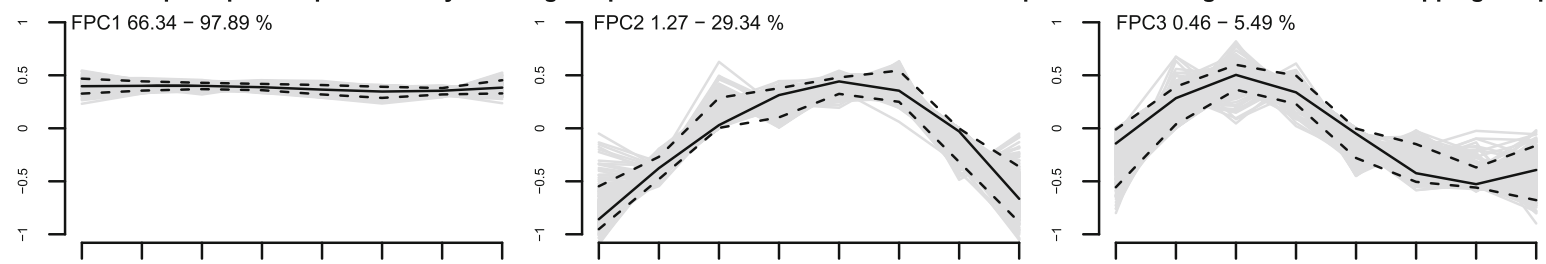

Wed Thu Fri Sat Sun Mon Tue Wed Wed Thu Fri Sat Sun Mon Tue Wed Wed Thu Fri Sat Sun Mon Tue Wed

F Functional principal component analysis using B-spline basis functions with individual-optimal smoothing and 1000 bootstrapping samples
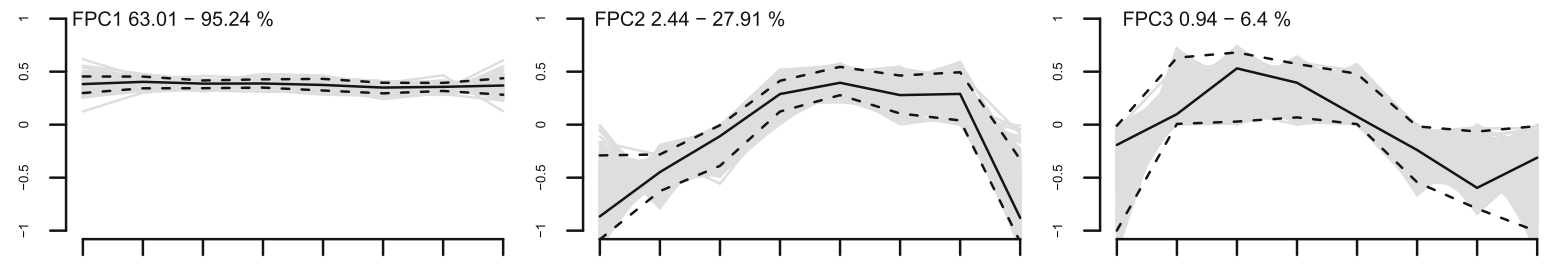

Wed Thu Fri Sat Sun Mon Tue Wed Wed Thu Fri Sat Sun Mon Tue Wed

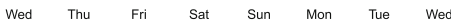

Fig. 3 (See legend on next page.) 
(See figure on previous page.)

Fig. 3 Bootstrapping confidence intervals (Cls) resulting from functional principal component analysis (FPCA) on 1000 re-samples obtained by a random sample with repetition from the original data sets. Panel $\mathbf{a}$ - Bootstrapping $\mathrm{Cl}$ resulting from a FPCA using Fourier basis functions and no smoothing parameter; Panel $\mathbf{b}$ - Bootstrapping $\mathrm{Cl}$ resulting from a FPCA using Fourier basis functions and common-optimal smoothing parameter; Panel $\mathbf{c}$ - Bootstrapping Cl resulting from a FPCA using Fourier basis functions and individual-optimal smoothing parameter; Panel $\mathbf{d}$ - Bootstrapping $\mathrm{Cl}$ resulting from a FPCA using B-splines basis functions and no smoothing parameter; Panel e - Bootstrapping Cl resulting from a FPCA using B-splines basis functions and common-optimal smoothing parameter; Panel $\mathbf{f}$ - Bootstrapping $\mathrm{Cl}$ resulting from a FPCA using B-splines basis functions and individual-optimal smoothing parameter 
A Functional principal component analysis using Fourier basis functions with no smoothing
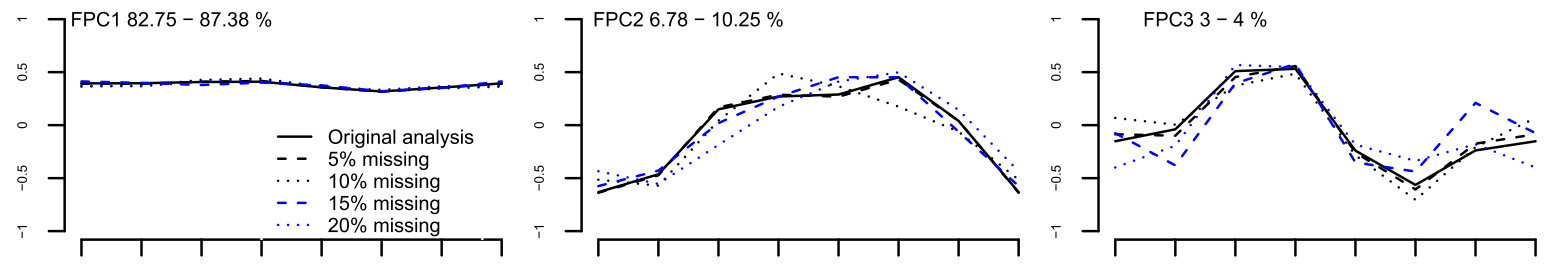

B. Wed Thu Fri Sat Sun Mon Tue Wed
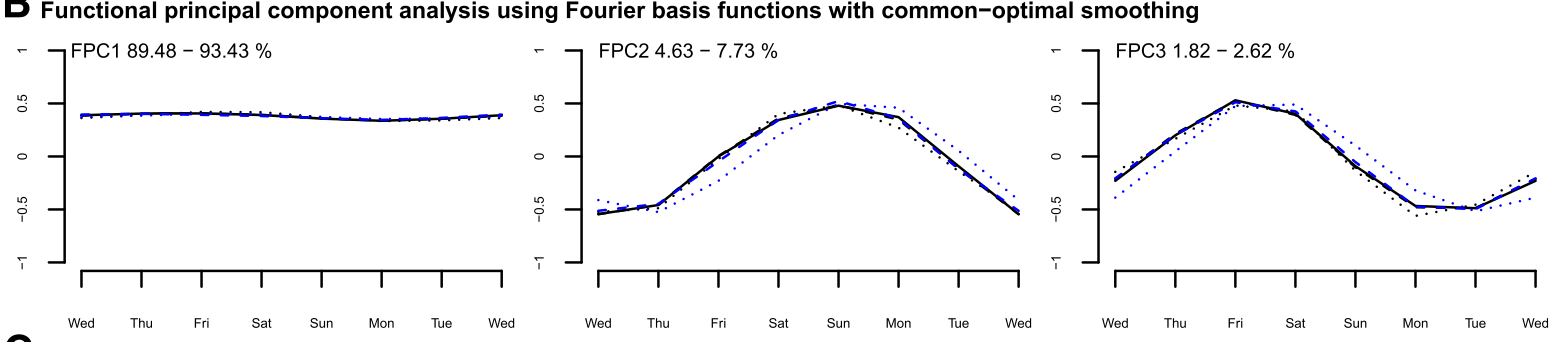

C Functional principal component analysis using Fourier basis functions with individual-optimal smoothing
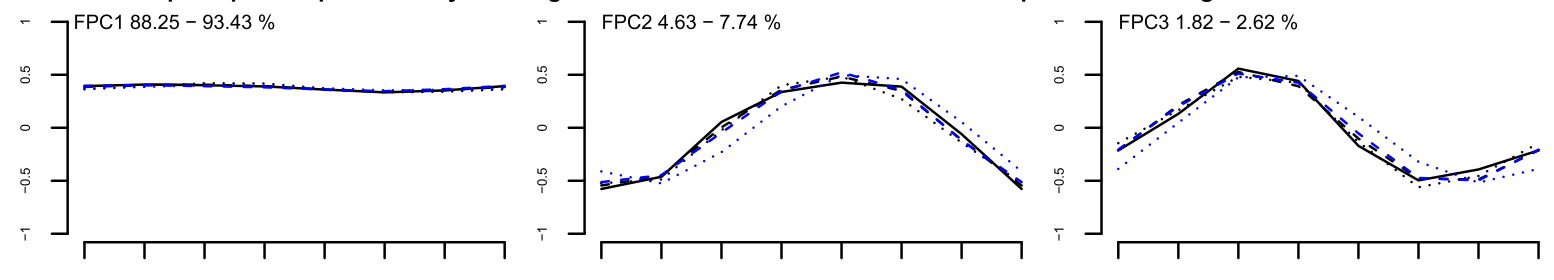

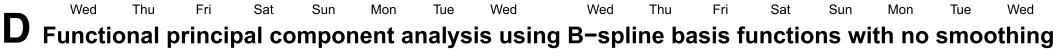
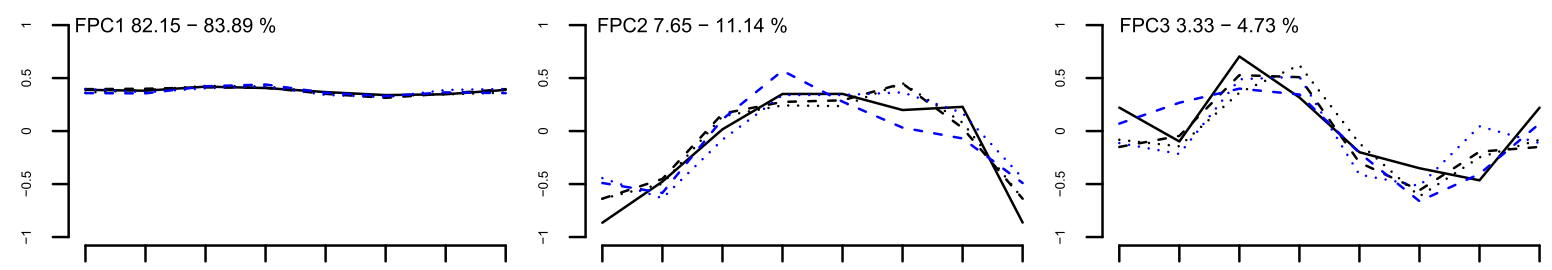

E Wed Thu Fri Sat Sun Mon Tue Wed
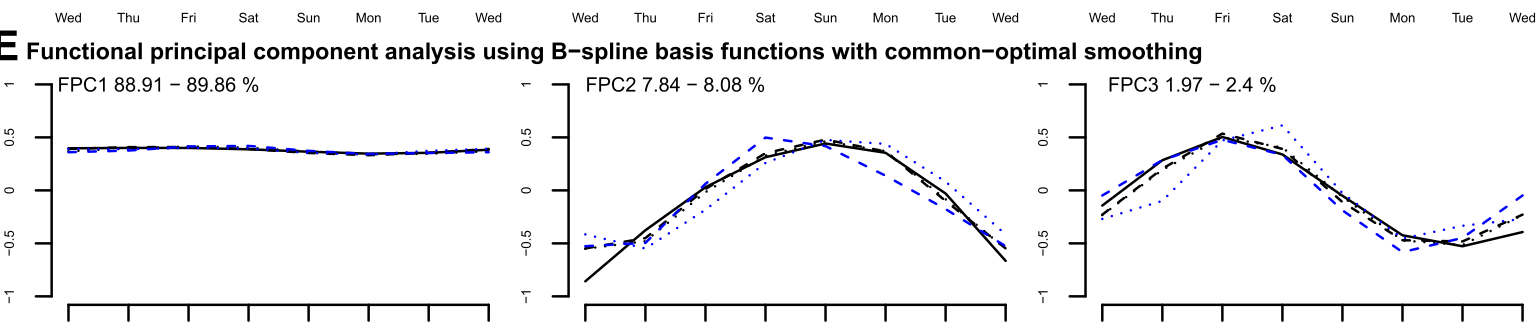

Wed Thu Fri sat Sun Mon Tue Wed Wed Thu Fri Sat Sun Mon Tue Wed

F Functional principal component analysis using B-spline basis functions with individual-optimal smoothing
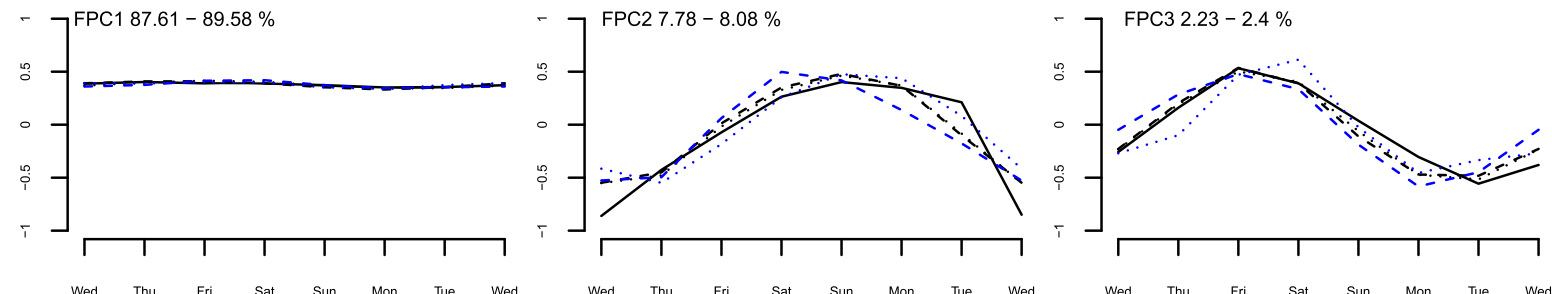

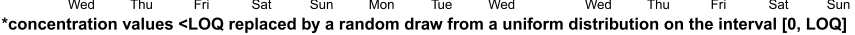

Fig. 4 (See legend on next page.) 
(See figure on previous page.)

Fig. 4 Sensitivity to missing for functional principal component analysis (FPCA) results. Panel a - Functional principal components (FPCS) resulting from a FPCA using Fourier basis functions and no smoothing parameter for 5, 10, 15, 20 \% of missing; Panel $\mathbf{b}$ - Functional principal components (FPCS) resulting from a FPCA using Fourier basis functions and common-optimal smoothing parameter for 5, 10, 15, 20 \% of missing; Panel c - Functional principal components (FPCs) resulting from a FPCA using Fourier basis functions and individual-optimal smoothing parameter for 5, 10, 15, 20 \% of missing; Panel $\mathbf{d}$ - Functional principal components (FPCs) resulting from a FPCA using B-splines basis functions and no smoothing parameter for 5, 10, 15, 20 \% of missing; Panel e - Functional principal components (FPCs) resulting from a FPCA using B-splines basis functions and common-optimal smoothing parameter for 5, 10, 15, $20 \%$ of missing; Panel $\mathbf{f}$ - Functional principal components (FPCS) resulting from a FPCA using B-splines basis functions and individual-optimal smoothing parameter for 5, 10, 15, $20 \%$ of missing 